\title{
Smart Starch-Gelatin Films Incorporated with Curcumin
}

\section{LE THI BICH NGUYET ${ }^{1}$ and VINH TIEN NGUYEN ${ }^{2 *}$}

'Science Research and Technology Transfer Institute, Industrial University of Ho Chi Minh City, Vietnam. ${ }^{2}$ Faculty of Chemical and Food Technology, Ho Chi Minh City University of Technology and Education, Vietnam.

*Corresponding author E-mail: tiennv@ @cmute.edu.vn

http://dx.doi.org/10.13005/ojc/360610

(Received: October 12, 2020; Accepted: December 05, 2020)

\begin{abstract}
S
In this study, we developed a starch-gelatin film incorporated with synthesized curcumin to be used as a $\mathrm{pH}$-sensitive smart material for food packaging. The film-forming mixture contained five components: starch, gelatin, glycerol, acetic acid and curcumin. The interactions of the components and their effects on the film properties were investigated by using response surface methodology with central composite experimental design. The results showed impacts of the contents of these components as independent variables on tensile strength, elongation at break, Young's modulus and solubility of the films. The contents of starch, gelatin and glycerol significantly affect these properties, while acetic acid and curcumin do not $(p<0.05)$. Also, it was shown that the incorporation of curcumin provided the film with the capacity to sense $\mathrm{pH}$ changes from neutral to basic (yellow at $\mathrm{pH} \leq 8$ and orange-red at $\mathrm{pH} \geq 9$ ).
\end{abstract}

Keywords: Smart packaging, Curcumin, pH indicator, Mechanical properties, Solubility.

\section{INTRODUCTION}

In recent years, the development of the food industry has created the need to develop smart food packaging films that are capable of evaluating the quality and safety of food during packaging, storage, transportation and distribution ${ }^{1}$. The $\mathrm{pH}$ of packed food items is a factor that changes due to their microbial spoilage during long term storage ${ }^{2}$. Hence, smart packaging films that can monitor the $\mathrm{pH}$ condition of micro-environment surrounding the packed food items have become an active and promising research area.
The main requirements for smart food packaging films are safety and biodegradability. The films should be made from materials of natural origin which do no harm to human health and do not pollute the food. In this respect, gelatin is a well-known candidate material thank to its good film-forming capacity, low costs, good transparency and biodegradability. However, one of the main disadvantages of gelatin films is that they have poor mechanical properties, high hygroscopicity and high solubility, therefore easily dissolve in contact with high-humidity food surfaces ${ }^{3}$.

This is an Open Access article licensed under a Creative Commons license: Attribution 4.0 International (CC- BY). Published by Oriental Scientific Publishing Company @ 2018 
Blending gelatin with starch is a commonlyused method to improve the physical properties and stability of the films. Starch-gelatin based films offer advantages in terms of mechanical properties as well as oxygen and water vapor barrier. Moreover, various types of colorimetric acid-base indicators were immobilized on biopolymeric matrices to enhance film properties. Musso et al., showed that gelatinbased films incorporated with synthetic or natural $\mathrm{pH}$ indicator such as bromocresol green, methyl orange, neutral red, curcumin and anthocyanins can change their color when contacting with liquids of various $\mathrm{pH}$. Besides, incorporating curcumin and starch into gelatin films enhances their mechanical, water vapor barrier and antioxidant properties ${ }^{5,6,7}$.

In this study, we produced edible and biodegradable composite films from starch, gelatin, glycerol, curcumin and acetic acid. Using the response surface methodology with central composite design, we studied the effects of these components on the mechanical and solubility of the films. We also evaluated the ability of the films containing curcumin to change their color with medium $\mathrm{pH}$ changes. The study would play a fundamental role for the further development of a $\mathrm{pH}$-sensitive smart packaging material.

\section{MATERIALS AND METHODS}

\section{Materials}

Corn starch was purchased from Rapunzel LTD. (Austria), granular gelatin from Himedia (India), acetic acid (99.9\%) and glycerol (99.9\%) from Xilong Scientific (China). Pure curcumin was synthesized according to a patent ${ }^{8}$ and purified by thrice recrystallization in $95 \%$ ethanol (Chemsol, Vietnam). Identity and purity of the synthesized curcumin were confirmed by NMR and MS techniques.

\section{EXPERIMENTAL}

The film-forming mixture contained 5 components: starch, gelatin, glycerol, acetic acid and curcumin. A half 5 -factor central composite experimental design was used to evaluate the effects of these components on the film properties. Our preliminary experiments determined the proper high and low values of each component content (Table 1). The composition of each film-forming mixture was then calculated using Minitab 15.0 software. The order of all experiments was randomized to reduce possibles time-dependent systematic errors.

Table 1: The boundary values of the factors

\begin{tabular}{cccc}
\hline Factor (content of film components, g) & Designation Low & High \\
\hline Starch & $\mathrm{x}_{1}$ & 0 & 9 \\
Gelatin & $\mathrm{x}_{2}$ & 0 & 9 \\
Glycerol & $\mathrm{x}_{3}$ & 3 & 5 \\
Acetic acid & $\mathrm{x}_{4}$ & 0 & 1.5 \\
Curcumin & $\mathrm{x}_{5}$ & 0 & 0.0225 \\
\hline
\end{tabular}

\section{Preparation of the composite films}

Precalculated amounts of film-forming components were blended in $100 \mathrm{~mL}$ of distilled water and then magnetically stirred under heating at $75^{\circ} \mathrm{C}$ for 30 minute. The solution was then cooled for $10 \mathrm{~min}$ poured into Petri dishes (10 g/dish), and left drying at room temperature for 48 hours. The films were removed from the dishes and stored in a desiccator with saturated solution $\mathrm{NaBr}$ (to maintain an air with $58 \%$ relative humidity) for at least $48 \mathrm{~h}$ to equilibrate the moisture content in the films.

\section{Characterization of the films}

The microstructural analysis of the films was carried out by using Scanning electronic microscope (SEM) (Hitachi S-4800, Japan) operating at a voltage of $10 \mathrm{kV}$. Fourier transform infrared (FTIR) spectra of the films were recorded with an ATR-FTIR spectrometer (Jasco FT/IR 4700, Germany) in the wavelength range from $4000 \mathrm{~cm}^{-1}$ to $400 \mathrm{~cm}^{-1}$ with a resolution of $4 \mathrm{~cm}^{-1}$ for 15 scans. The film color was determined using a colorimeter (Minolta, Japan).

The tensile strength (TS), elongation at break (EB) and Young's modulus (YM) of the films were determined using a tensile tester (Testometric, UK) according to the ASTM Standard method D88212. The films were cut into rectangles of $70 \times 30 \mathrm{~mm}^{2}$. Initial separation of the grips was $40 \mathrm{~mm}$ and the crosshead speed was $0.8 \mathrm{~mm} / \mathrm{s}$. The tensile test was replicated 5 times for each film sample.

TS of the films (MPa) was calculated as follows:

$T S=\frac{N_{\max }}{A}$

Where $N_{\max }$ is the maximum load $(\mathrm{N})$ applied to break the film sample and $A$ is the area (thickness $\times$ width, $\mathrm{mm}^{2}$ ) of the film cross-section. 
Before tensile tests, the thickness of each film was measured at 10 points using a thickness meter (Mitutoyo, Japan).

EB of the films (\%) was calculated as follows:

$$
E B=\frac{l-l_{o}}{l_{o}} \times 100
$$

Where $/$ is the length $(\mathrm{mm})$ of the film when broken and $\mathrm{I}_{0}=40 \mathrm{~mm}$ is the initial grip separation. as follows:

Finally, YM of the films (MPa) was calculated

$$
Y M=\frac{\text { Stress }}{\text { Strain }}
$$

Where Stress $\left(\mathrm{N} / \mathrm{mm}^{2}\right)$ is the maximum load (N) divided by cross-section area $\left(\mathrm{mm}^{2}\right)$ and Strain is the change in length $(\mathrm{I}-\mathrm{I}, \mathrm{mm})$ divided by original length $\left(I_{0}=40 \mathrm{~mm}\right)$.

To determine the film solubility, each film was cut into $2 \times 2-\mathrm{cm}^{2}$ squares, dried in an oven at $105^{\circ} \mathrm{C}$ for $24 \mathrm{~h}$ and weighed to obtain the mass of dry matter $(\mathrm{m0}, \mathrm{g})$. The dried film was then immersed in distilled water for $2 \mathrm{~h}$, dried again at $105^{\circ} \mathrm{C}$ for 24 $\mathrm{h}$ and weighed to obtain the remaining dry weight $\left(m_{1}, g\right)$. All solubility experiments were conducted in triplicate. The solubility of the films S (\%) was calculated as follows:

$$
S=\frac{m_{o}-m_{1}}{m_{o}} \times 100
$$

\section{Responses of the films to $\mathrm{pH}$ changes}

The film containing no acetic acid was used to investigate its response to $\mathrm{pH}$ change. Each film sample was wetted with 3 drops of a phosphate buffer ( $\mathrm{pH}$ from 4 to 11). The film color before and after getting in contact with the buffer was determined using a colorimeter at 10 random points. The $L^{*}{ }^{*} b$ color scale was used to express the degree of lightness $(\mathrm{L})$, redness - greenness (a), and yellowness - blueness (b) of the films. The total color difference $(\Delta \mathrm{E})$, compared to the white standard associated with the colorimeter, was defined as follows:

$$
\Delta E=\sqrt{(\Delta L)^{2}+(\Delta a)^{2}+(\Delta b)^{2}}
$$

\section{RESULTS AND DISCUSSION}

\section{FTIR Analysis}

FTIR spectroscopy was used to characterize the interaction between gelatin and starch. The FTIR spectra for gelatin, starch and composite film are shown in Fig. 1 in the range of $4000-400 \mathrm{~cm}^{-1}$. For the reader's convenience, the positions of all the peaks were noted explicitly in Fig. 1. However, we would discuss below only some characteristic peaks.

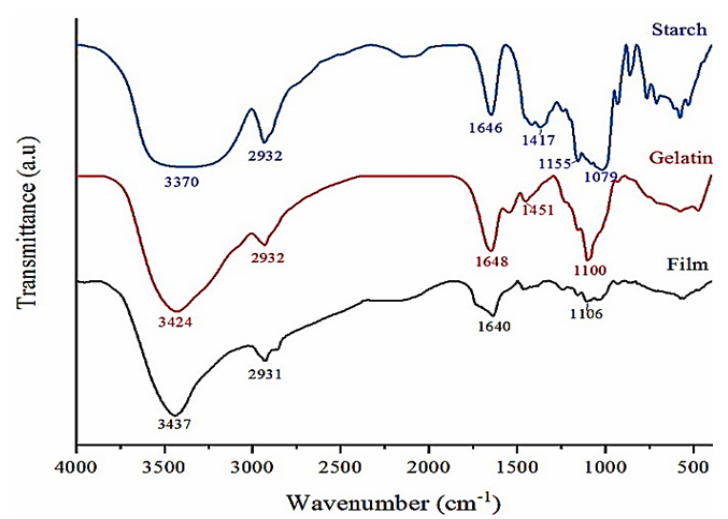

Fig. 1. FTIR spectra of gelatin, starch and film

In Fig. 1, the broad peak at $3370 \mathrm{~cm}^{-1}$ for starch and the sharp peak at $3424 \mathrm{~cm}^{-1}$ for gelatin are assignable to $-\mathrm{OH}$ and $-\mathrm{NH}$ stretching vibrations, respectively. The peak at $2931 \mathrm{~cm}^{-1}$ (film) and the other two at $2932 \mathrm{~cm}^{-1}$ (starch and gelatin spectra) are assignable to $\mathrm{C}-\mathrm{H}$ stretching. $\mathrm{C}=\mathrm{O}$ stretching was observed via the peaks at $1646 \mathrm{~cm}^{-1}$ (starch), $1648 \mathrm{~cm}^{-1}$ (gelatin) and $1640 \mathrm{~cm}^{-1}$ (film). The peak at $1100 \mathrm{~cm}^{-1}$ for gelatin represents its characteristic C-N stretching. Finally, the peaks at $1079 / 1155 \mathrm{~cm}^{-1}$ for starch and $1106 \mathrm{~cm}^{-1}$ for the film represent C-O stretching vibration. The characteristic peaks were summarized in Table 2.

Table 2: Characteristic peaks from FTIR spectra of starch, gelatin and the film

\begin{tabular}{lccc}
\hline $\begin{array}{l}\text { Characteristic } \\
\text { Gelatin }\end{array}$ & Starch & Film & Characteristic group \\
\hline 1100 & - & - & C-N stretching \\
2932 & 2932 & 2931 & C-H stretching \\
1451 & 1417 & - & C-H bending \\
1648 & 1646 & 1640 & C=O stretching \\
- & $1079 / 1155$ & 1106 & C-O stretching \\
3424 & - & 3437 & $-\mathrm{NH}$ stretching \\
- & 3370 & - & $-\mathrm{OH}$ stretching \\
\hline
\end{tabular}

Chemical interactions of the film components are reflected by changes in the peaks of FTIR spectra. For example, in the spectrum of the composite film, the peaks originally related to $-\mathrm{OH}$ and $-\mathrm{NH}$ stretching vibrations in starch and gelatin, respectively, shifted from $3424 \mathrm{~cm}^{-1}$ and 
$3370 \mathrm{~cm}^{-1}$ to $3437 \mathrm{~cm}^{-1}$. This change demonstrates the intermolecular hydrogen bonds between amino groups of gelatin and hydroxyl groups of starch ${ }^{9}$.

\section{SEM Analysis}

The SEM microphotographs of the composite film using magnifications of $\times 200$ and $\times 5000$ are shown in Figure 2.

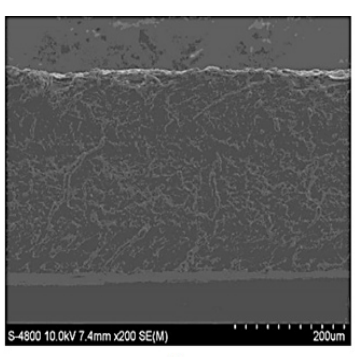

(a)

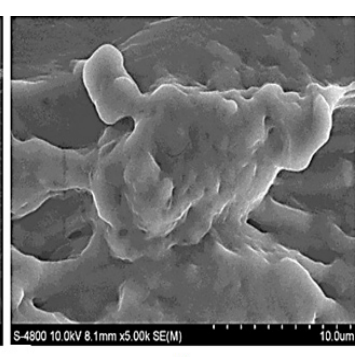

(b)
Fig. 2. SEM images of the film cross-section with magnifications of $\times 200$ (a) and $\times 5000$ (b)

The SEM image at $\times 200$ showed that the structure of the film was homogeneous and dense (Fig. 2a). At $\times 5000$, the presence of pores/cavities in the structure was observed (Fig. 2b). Corn starch is composed of about $28 \%$ of amylose and about $72 \%$ of amylopectin. Amylose is a linear chain of glucose units linked with $\alpha-(1 \rightarrow 4)$ glycosidic bonds. Meanwhile, amylopectin is a chain of linear $\alpha$ - $(1 \rightarrow 4)$-linked glucose units branched with $\alpha-(1 \rightarrow 6)$ - branch points ${ }^{10}$. The arrangement of starch and gelatin during mixing and drying process might leave intergranular spaces relating to the formation of pores/cavities.

\section{Study of mechanical properties and solubility}

Formulations of the composite films were designed by using CCD-RSM. The results for mechanical properties and solubility of the film samples are presented in Table 3, together with the corresponding film formulations.

Table 3: Tensile strength (TS), elongation at break (EB), young modulus (YM) and solubility (S) of the films

\begin{tabular}{|c|c|c|c|c|c|c|c|c|c|}
\hline \multirow[t]{2}{*}{ Design point } & \multicolumn{4}{|c|}{ Independent variables } & \multicolumn{5}{|c|}{ Dependent variables* } \\
\hline & $x_{1}$ & $x_{2}$ & $x_{3}$ & $x_{4}$ & $x_{5}$ & TS (MPa) & $\mathrm{EB}(\%)$ & YM (MPa) & $\mathrm{S}(\%)$ \\
\hline 1 & 6 & 6 & 4 & 1 & 0.015 & $1.502 \pm 0.044$ & $71.755 \pm 3.784$ & $12.064 \pm 0.515$ & 43.90 \\
\hline 2 & 12 & 6 & 4 & 1 & 0.015 & $3.506 \pm 0.418$ & $55.270 \pm 10.440$ & $83.682 \pm 19.448$ & 15.22 \\
\hline 3 & 6 & 6 & 4 & 1 & 0.015 & $1.494 \pm 0.261$ & $83.455 \pm 18.650$ & $10.106 \pm 4.363$ & 45.45 \\
\hline 4 & 3 & 9 & 3 & 0.5 & 0.0075 & $5.182 \pm 1.697$ & $120.051 \pm 12.460$ & $47.026 \pm 10.609$ & 30.77 \\
\hline 5 & 6 & 0 & 4 & 1 & 0.015 & $0.688 \pm 0.165$ & $30.059 \pm 12.789$ & $4.731 \pm 0.475$ & 56.67 \\
\hline 6 & 9 & 9 & 5 & 0.5 & 0.0075 & $2.652 \pm 0.207$ & $84.766 \pm 6.984$ & $41.014 \pm 14.319$ & 44.19 \\
\hline 7 & 9 & 9 & 3 & 1.5 & 0.0075 & $6.876 \pm 1.469$ & $51.979 \pm 4.987$ & $190.118 \pm 59.459$ & 47.87 \\
\hline 8 & 6 & 6 & 4 & 1 & 0.015 & $1.896 \pm 0.095$ & $74.662 \pm 4.988$ & $13.202 \pm 1.126$ & 48.39 \\
\hline 9 & 3 & 9 & 3 & 1.5 & 0.0225 & $3.759 \pm 0.242$ & $144.732 \pm 9.652$ & $22.575 \pm 1.551$ & 53.93 \\
\hline 10 & 9 & 3 & 3 & 1.5 & 0.0225 & $4.472 \pm 0.307$ & $51.733 \pm 5.040$ & $129.317 \pm 31.679$ & 43.75 \\
\hline 11 & 6 & 12 & 4 & 1 & 0.015 & $4.489 \pm 0.793$ & $106.772 \pm 12.064$ & $55.841 \pm 12.064$ & 29.67 \\
\hline 12 & 6 & 6 & 2 & 1 & 0.015 & $7.922 \pm 0.920$ & $29.348 \pm 20.832$ & $314.442 \pm 67.569$ & 20.83 \\
\hline 13 & 0 & 6 & 4 & 1 & 0.015 & $4.405 \pm 0.302$ & $78.181 \pm 3.010$ & $56.002 \pm 13.941$ & 47.06 \\
\hline 14 & 3 & 3 & 3 & 1.5 & 0.0075 & $0.336 \pm 0.071$ & $36.405 \pm 20.825$ & $12.550 \pm 12.413$ & 59.14 \\
\hline 15 & 9 & 9 & 3 & 0.5 & 0.0225 & $8.275 \pm 0.393$ & $45.566 \pm 13.426$ & $236.701 \pm 66.806$ & 22.11 \\
\hline 16 & 3 & 9 & 5 & 0.5 & 0.0225 & $1.210 \pm 0.166$ & $118.595 \pm 12.960$ & $3.606 \pm 0.355$ & 48.98 \\
\hline 17 & 6 & 6 & 4 & 1 & 0.015 & $1.551 \pm 0.157$ & $71.427 \pm 21.448$ & $11.637 \pm 0.908$ & 43.00 \\
\hline 18 & 3 & 9 & 5 & 1.5 & 0.0075 & $1.671 \pm 0.059$ & $165.813 \pm 7.920$ & $5.143 \pm 2.155$ & 46.15 \\
\hline 19 & 6 & 6 & 4 & 1 & 0 & $1.684 \pm 0.104$ & $84.754 \pm 19.209$ & $12.504 \pm 3.456$ & 52.13 \\
\hline 20 & 3 & 3 & 3 & 0.5 & 0.0225 & $0.349 \pm 0.028$ & $41.345 \pm 1.271$ & $3.115 \pm 1.972$ & 53.85 \\
\hline 21 & 6 & 6 & 4 & 1 & 0.015 & $1.609 \pm 0.289$ & $62.827 \pm 8.234$ & $19.521 \pm 6.939$ & 45.00 \\
\hline 22 & 9 & 3 & 5 & 1.5 & 0.0075 & $1.314 \pm 0.102$ & $33.994 \pm 6.312$ & $8.701 \pm 1.864$ & 23.33 \\
\hline 23 & 9 & 3 & 3 & 0.5 & 0.0075 & $2.085 \pm 0.474$ & $32.114 \pm 10.067$ & $35.481 \pm 9.507$ & 35.48 \\
\hline 24 & 6 & 6 & 4 & 1 & 0.015 & $1.379 \pm 0.249$ & $69.356 \pm 12.195$ & $7.819 \pm 1.972$ & 41.03 \\
\hline 25 & 6 & 6 & 4 & 1 & 0.03 & $1.732 \pm 0.203$ & $89.834 \pm 12.460$ & $12.386 \pm 3.750$ & 47.31 \\
\hline 26 & 9 & 9 & 5 & 1.5 & 0.0225 & $1.167 \pm 0.129$ & $73.991 \pm 14.866$ & $8.241 \pm 0.374$ & 51.06 \\
\hline 27 & 9 & 3 & 5 & 0.5 & 0.0225 & $0.932 \pm 0.010$ & $26.683 \pm 2.394$ & $6.359 \pm 0.271$ & 19.28 \\
\hline 28 & 6 & 6 & 4 & 2 & 0.015 & $1.288 \pm 0.128$ & $94.886 \pm 16.675$ & $6.943 \pm 1.197$ & 32.14 \\
\hline 29 & 6 & 6 & 4 & 0 & 0.015 & $0.920 \pm 0.379$ & $88.067 \pm 14.502$ & $4.874 \pm 1.174$ & 43.18 \\
\hline 30 & 3 & 3 & 5 & 1.5 & 0.0225 & $0.254 \pm 0.063$ & $17.590 \pm 2.866$ & $6.487 \pm 1.438$ & 48.94 \\
\hline 31 & 6 & 6 & 6 & 1 & 0.015 & $0.469 \pm 0.097$ & $80.816 \pm 40.224$ & $2.584 \pm 0.158$ & 64.13 \\
\hline 32 & 3 & 3 & 5 & 0.5 & 0.0075 & $0.404 \pm 0.078$ & $37.171 \pm 11.509$ & $4.891 \pm 2.100$ & 65.96 \\
\hline
\end{tabular}

*The results are expressed as mean \pm standard deviation $(n=3)$. 
Using regression analysis for the data from the CCD experiments, a second-order empirical model was developed:

$$
\begin{aligned}
& Y=b_{0}+b_{1} x_{1}+b_{2} x_{2}+b_{3} x_{3}+b_{4} x_{4}+b_{5} x_{5} \\
& +b_{12} x_{1} x_{2}+b_{13} x_{1} x_{3}+b_{14} x_{1} x_{4}+b_{15} x_{1} x_{5} \\
& +b_{23} x_{2} x_{3}+b_{24} x_{2} x_{4}+b_{25} x_{2} x_{5}+b_{34} x_{3} x_{4}+b_{35} x_{3} x_{5} \\
& +b_{45} x_{4} x_{5}+b_{11} x_{1}^{2}+b_{22} x_{2}^{2}+b_{33} x_{3}^{2}+b_{44} x_{4}^{2}+b_{55} x_{5}^{2}
\end{aligned}
$$

Where Y-response variables, including TS, EB, YM and solubility; $x_{1}, x_{2}, x_{3}, x_{4}, x_{5}-$ independent variables, which are the contents of starch, gelatin, glycerol, acetic acid and curcumin, respectively; $b_{0}$-intercept; $b_{1}, b_{2}, b_{3}, b_{4}$, $b_{5}$ - regression coefficients for linear terms; $b_{12}$, $b_{13}, b_{14}, b_{15}, b_{23}, b_{24}, b_{25}, b_{34}, b_{35}, b_{45}$-regression coefficients for interactions; $b_{11}, b_{22}, b_{33}, b_{44}$, $b_{55}$-regression coefficients for quadratic terms. Regression coefficients for each term and their $p$-values from the analysis of variance are shown in Table 4.

Table 4: Regression coefficients and analysis of variance of the second-order polynomials for TS, EB, YM and solubility $S$

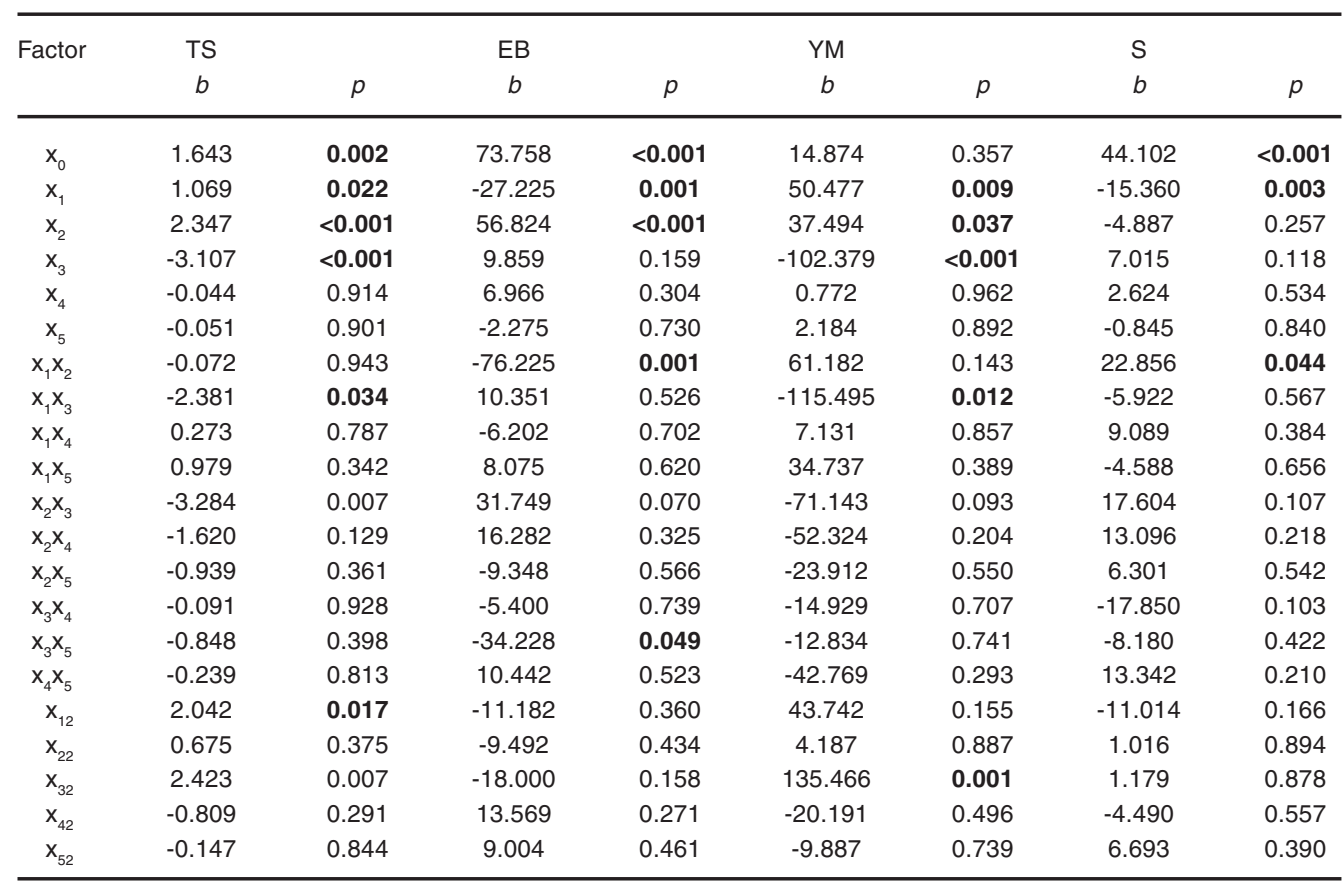

The coefficients of determinations $\left(R^{2}\right)$ were 0.9276 for TS, 0.9282 for $\mathrm{EB}, 0.9019$ for YM and 0.785 for solubility. These numbers mean that $92.76 \%$, $92.82 \%, 90.19 \%$ and $78.5 \%$ of the experimental data can be explained by the fitted equations for TS, EB, YM and solubility, respectively. The high values of $\mathrm{R}^{2}$ indicate the fidelity of the adopted equations in modelling the effects of the composition of the films on their mechanical properties and solubility.

To visualize the revealed interactions between the independent variables on the responses, response surface plots were constructed for those pairs of independent variables that showed significant interactions $(p<0.05)$ (Fig. 3). The remaining variables were kept constant at their middle values.
The results in Table 4 show that TS of the films is mainly affected by varying the content of the polymers and glycerol $(p<0.05)$. TS values varied between 0.254 and $8.275 \mathrm{MPa}$ (Table 3), with minimum and maximum with the starchgelatin-glycerol combination of $(3,3,5)$ and $(9$, 9 , 3) (gram), respectively (see also Fig. 3a,b). It is well known that the hydroxyl groups in starch form intra- and intermolecular hydrogen bonds with each other. The same happens between the amino groups in gelatin. When these two polymers are blended, additional hydrogen bonds appear between the hydroxyl and the amino groups, enhancing mechanical stability of the films ${ }^{11}$. Our results are in agreement with a previous research ${ }^{12}$, in which TS of films from chicken-skin gelatin was improved when incorporated with starch. 

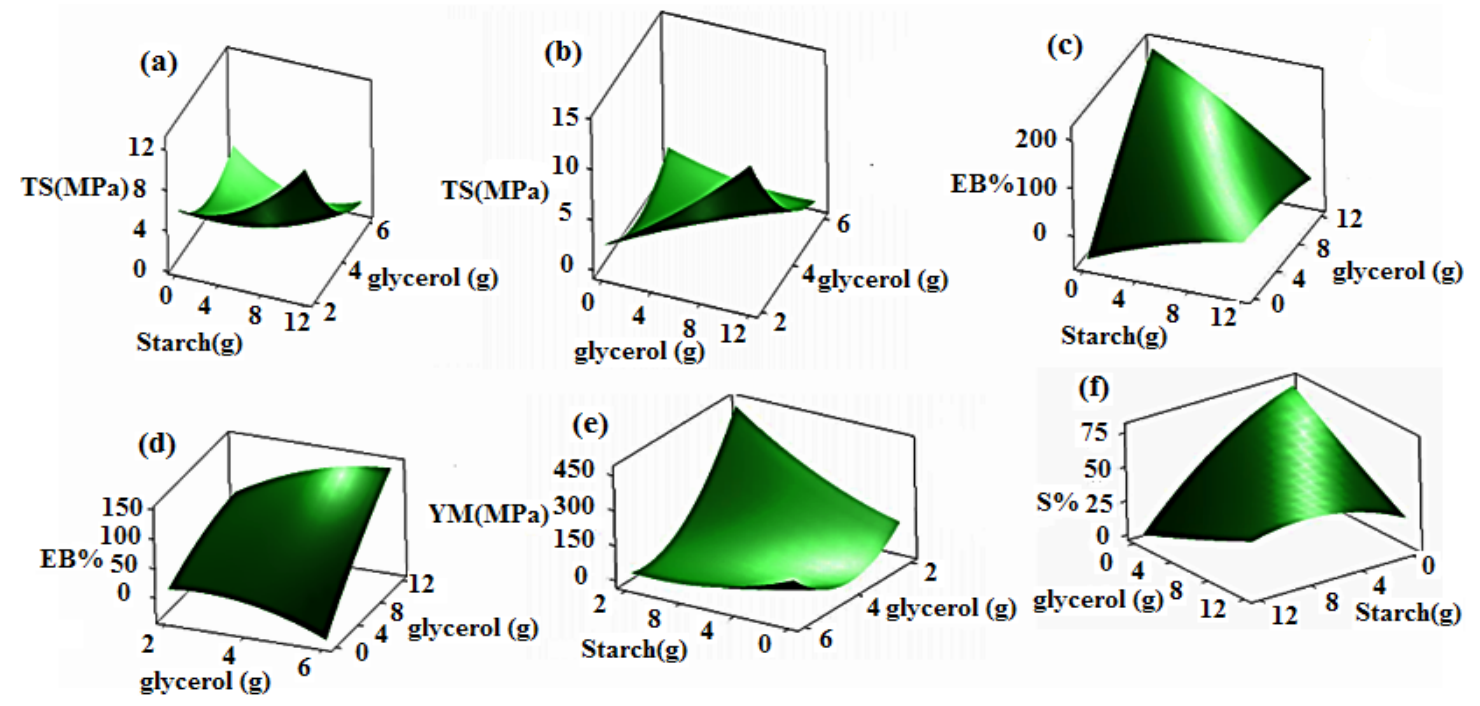

Fig. 3. Response surface plots showing the interactions of independent variables on TS (a, b), EB (c, d), YM (e) and S (f)

Glycerol also had a significant effect on TS of the films $(p<0.001)$ (Table 4). Fig. $2(a, b)$ showed that at low levels of starch and gelatin, TS increased with the increase in glycerol concentration. In contrast, at high levels of starch and gelatin, higher glycerol concentration led to lower TS. Glycerol molecules are small and hydrophilic with three hydroxyl groups, thus can lie between hydrophilic polymer chains and acts as a plasticizer ${ }^{4}$. By forming hydrogen bonds with starch and gelatin molecules, glycerol diminish the direct hydrogen bonds between the polymer chains, thus reducing $\mathrm{TS}^{11}$.

Regarding EB, both starch and gelatin were found to have profound effects on the elongation property of the films $(p<0.05)$. EB values varied between 17.590 and $165.813 \%$ (Table 3) and were larger when higher gelatin concentration was applied (Fig. 3c, d). At high levels of gelatin, EB decreased with the increase in starch content (Fig. 3c). The decrease in $E B$ is possibly due to strong intermolecular interactions between the chains of starch and gelatin, which reduces flexibility of the films. In contrast, at low levels of gelatin, EB slightly increased with the increase in starch concentration (Fig. 3c). The observed increase in EB was related to the change in mobility of polymeric chains ${ }^{15}$. The plasticizer reduces the interand intramolecular bonds between molecules of starch and gelatin, and then substitutes these bonds with hydrogen bonds formed between it and the polymeric molecules. Such disruption and reconstruction of starch and gelatin chains promote film flexibility and allow more chain mobility ${ }^{13}$. Moreover, glycerol with hydrophilic nature can hold more water molecules. The absorbed moisture could cause an extra plasticizing effect that led to the increase in EB and decrease in TS of the films ${ }^{13,14}$.

YM measures the stiffness of materials: a higherYM indicates a stiffer film ${ }^{12}$. The positive main effects of starch and gelatin mean that increasing the content of these polymers resulted in stiffer films (Table 4). This effect can be explained again by the strong intermolecular interaction between the chains of starch and gelatin, which produces a stronger structure of the films (Figure 3e).

Finally, the water solubilities of the films are shown in Table 3. The content of starch significantly affects the film solubility $(p<0.05)$. Fig. $3 f$ shows that the highest solubility $(65.96 \%)$ was observed in the film with $3 \mathrm{~g}$ of starch, while the lowest solubility $(15.22 \%)$ in the film with $12 \mathrm{~g}$ of starch. This change might be due to the intermolecular interactions between the chains of starch and gelatin, which reduces the number of available functional groups such as $-\mathrm{OH}$ and $-\mathrm{NH}_{2}$ that can form hydrogen bonds with water. Moreover, the strong interaction between starch and gelatin might enhance film cohesiveness, thus preventing the diffusion of water into the film. These observations are supported by a previous study where the film solubility decreased as starch was blended with gelatin at higher content ${ }^{12}$.

\section{pH-sensitivity}

In this study, we used curcumin as a 
sustainable and safe indicator for smart packaging materials. It is well-known that curcumin changes its color from yellow to orange-red when the medium $\mathrm{pH}$ changes to alkaline due to the reaction with $\mathrm{OH}$ - group ${ }^{16}$.

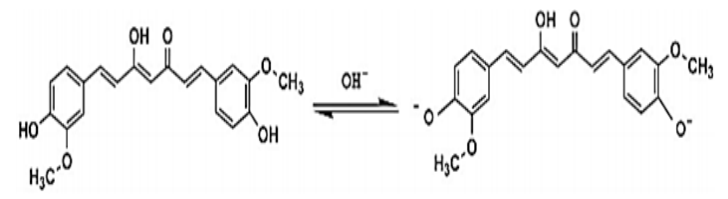

Scheme 1. Reaction of curcumin with $\mathrm{OH}$ - group

The test film was homogeneously yellow due to the uniform dispersion of curcumin. Fig. 4 shows that its yellow color did not change when getting in contact with acidic-neutral media $(\mathrm{pH} \leq 8)$, but turned red-orange in contact with alkaline media $(\mathrm{pH} \geq 9)$. Moreover, the stronger the alkaline media was, the more intensive the orange-red coloration became.

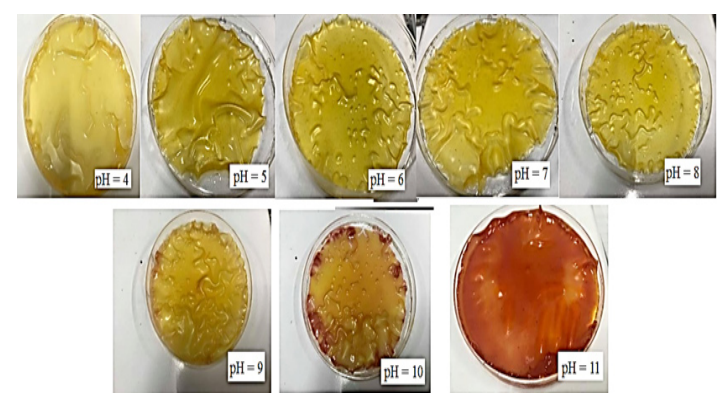

Fig. 4. Responses of the film when getting contact with buffers of different $\mathrm{pH}$

The parameters $L, a, b$ and $\Delta E$ indicating the film colors are shown in Table 5. The coordinate a represents a color between red (positive a) and green (negative $a$ ) and $b$ represent a color between yellow (positive $b$ ) and blue (negative $b$ ). The parameter $L$ represents the brightness, ranging from 0 (dark) to 100 (bright). Color changes with $\Delta \mathrm{E}$ higher than 5 can be detected easily by human eyes, and $\Delta \mathrm{E}$ higher than 12 indicates an obvious change in color $^{17}$. When the buffer $\mathrm{pH}$ was in the range of $4-8$, the values of $L$ (from 71 to 76 ), a (from -0.5 to -5.7 ) and $b$ (from 97.5 to 71.3 ) can be interpreted as a bright yellow color with a very small shade of green, which is in accordance with the upper panel of Fig. 4. Looking closer at the values of $\Delta E$, one could see that there was a small color change when $\mathrm{pH}$ increased from 4 to 5 , then the color remained quite the same at $\mathrm{pH}$ ranging from 5 to 8 .

However, when $\mathrm{pH}$ increased to 9 and higher, all color parameters varied significantly, indicating distinct color changes. To be more specific, a strong reduction of $L$ is understood as that the color became darker; an increase of a means an increase of redness; and a small reduction of $b$ means that yellowness slightly decreased, which can be seen in the lower panel of Fig. 4. Strong color changes occurred when $\mathrm{pH}$ increased from 8 to 9 , and then from 10 to 11 . These results demonstrated that the starch-gelatin films incorporated with curcumin can be applied as a smart packaging material, which can inform consumers about the product quality change, which associates with a $\mathrm{pH}$ change from neutral to basic ${ }^{6}$.

Table 5: The color parameters ( $L$, a and $b$ ) and total color different $(\Delta E)$ of the films in contact with solutions with various $\mathrm{pH}$

\begin{tabular}{|c|c|c|c|c|c|c|c|c|}
\hline $\mathrm{pH}$ & 4 & 5 & 6 & 7 & 8 & 9 & 10 & 11 \\
\hline $\mathrm{L}$ & $71.3 \pm 2.0$ & $71.0 \pm 1.0$ & $74.4 \pm 0.4$ & $76.1 \pm 0.5$ & $74.6 \pm 1.6$ & $68.4 \pm 0.9$ & $58.2 \pm 1.4$ & $46.8 \pm 0.4$ \\
\hline a & $-0.5 \pm 0.1$ & $-2.3 \pm 0.5$ & $-3.6 \pm 0.5$ & $-2.1 \pm 0.2$ & $-5.7 \pm 0.5$ & $6.9 \pm 3.3$ & $19.5 \pm 2.2$ & $70 \pm 0.6$ \\
\hline b & $79.5 \pm 2.9$ & $72.8 \pm 0.5$ & $74.6 \pm 2.1$ & $77.9 \pm 0.6$ & $71.3 \pm 1.2$ & $65.7 \pm 2.6$ & $60 \pm 1.9$ & $60 \pm 1.3$ \\
\hline$\Delta \mathrm{E}$ & $79.5 \pm 3.2$ & $72.4 \pm 0.7$ & $74.5 \pm 2.1$ & $77.3 \pm 0.5$ & $71.6 \pm 3.5$ & $65.3 \pm 2.7$ & $65.4 \pm 2.9$ & $98.5 \pm 1.4$ \\
\hline
\end{tabular}

\section{CONCLUSION}

A promising smart food packaging film based on starch-gelatin incorporated with curcumin was investigated. The SEM micrograph of the film cross-section showed a homogeneous and compact structure. The film's capacity to sense media $\mathrm{pH}$ was tested. As a food packaging material, the film could provide information about the quality of products by changing its color. RMS-CCD was successfully applied to investigate the effects of the film components on its properties. The results demonstrated that the examined responses for physical and mechanical properties were strongly influenced not only by starch, gelatin and glycerol contents separately but also by their interactions. This observation opened the window to further studies with the aim of finding the optimized formulation which provides the best physical properties for the composite film. 


\section{ACKNOWLEDGEMENT}

The authors are grateful to Science Research and Technology Transfer Institute, Industrial University of Ho Chi Minh City and Faculty of Chemical and Food Technology, Ho Chi Minh City University of Technology and Education. We thank
Dr. Chien-Thang Tran for careful reading of the manuscript and insightful comments.

\section{Conflict of interest}

The authors declare that there is no conflict of interest.

\section{REFERENCES}

1. Mustafa, F.; Andreescu, S. Foods., 2018 7(10), 168-188.

2. Rawat, S. Asian J. Plant Sci. Res., 2015, 5(4), 47-56.

3. Rattaya, S.; Benjakul, S.; Prodpran, T. J. Food Eng., 2009, 95(1), 151-157.

4. Ramos, M.; Valdes, A.; Beltran, A.; Garrigós, M. C. Coatings., 2016, 6(4), 41-61.

5. Musso, Y. S.; Salgado, P. R.; Mauri, A. N. Food Hydrocoll., 2016, 61, 523-530.

6. Musso, Y. S.; Salgado, P. R.; Mauri, A. N. Food Hydrocoll., 2017, 66(8), 8-15.

7. Musso, Y. S.; Salgado, P. R.; Mauri, A. N. Food Hydrocoll., 2019, 89, 674-681.

8. Pabon, H. J. J. Rec. Trav. Chim. Pays Bas., 1964, 83(4), 379-386.

9. Zhong, Q. P.; Xia, W. S. Food Technol. Biotechnol., 2008, 46(3), 262-269.

10. Jha, P.; Dharmalingam, K.; Nishizu, T.; Katsuno, N.; Anandalakshmi, R. Starch-
Stärke., 2020, 72(1-2), 1900121-1900130.

11. Thakur, R.; Saberi, B.; Pristijono, P.; Stathopoulos, C. E.; Golding, J. B.; Scarlett, C. J.; Bowyer, M.; Vuong, Q. V. J. Food Sci. Technol., 2017, 54(8), 2270-2278.

12. Alias, S. A.; Sarbon, N. M. NPJ Sci. Food., 2019, 3(1), 1-9.

13. Sanyang, M. L.; Sapuan, S. M.; Jawaid, M.; Ishak, M. R.; Sahari, J. Polymers., 2015, 7(6), 1106-1124.

14. Chinma, C. E.; Ariahu, C. C.; Alakali, J. S. J. Food Sci. Technol., 2015, 52(4), 2380-2386.

15. da Rosa Zavareze, E.; Pinto, V. Z.; Klein, B.; El Halal, S.L.; Elias, M.C.; Prentice-Hernández, C.; Dias, A. R. Food Chem., 2012, 132(1), 344-50.

16. Ma, Q.; Du, L.; Wang, L. Sens. Actuators B Chem., 2017, 244, 759-766.

17. Liang, T.; Sun, G.; Cao, L.; Li, J.; Wang, L. Food Hydrocoll., 2019, 87, 858-868. 